\title{
Trions in coupled quantum wells and Wigner crystallization
}

\author{
Oleg L. Berman ${ }^{1,2}$, Roman Ya. Kezerashvili ${ }^{1,2}$, and Shalva M. Tsiklauri ${ }^{1}$ \\ ${ }^{1}$ Physics Department, New York City College of Technology, The City University of New York, \\ Brooklyn, NY 11201, USA \\ ${ }^{2}$ The Graduate School and University Center, The City University of New York, \\ New York, NY 10016, USA
}

(Dated: October 30, 2018)

\begin{abstract}
We consider a restricted three body problem, where two interacted particles are located in two dimensional (2D) plane and interact with the third one located in the parallel spatially separated plane. The system of such type can be formed in the semiconductor coupled quantum wells, where the electrons (or holes) and direct excitons spatially separated in different parallel neighboring quantum wells that are sufficiently close to interact and form negative $X^{-}$or positive $X^{+}$indirect trions. It is shown that at large interwell separations, when the interwell separation much greater than the exciton Bohr radius, this problem can be solved analytically using the cluster approach. Analytical results for the energy spectrum and the wave functions of the spatially indirect trion are obtained, their dependence on the interwell separations is analyzed and a conditional probability distribution is calculated. The formation of $2 \mathrm{D}$ Wigner crystal of trions at the low densities is predicted. It is shown that the critical density of the formation of the trion Wigner crystal is sufficiently greater than the critical density of the electron Wigner crystal.

PACS numbers: 71.35.Pq, 71.35.-y, 73.21.Fg
\end{abstract}

\section{INTRODUCTION}

The linear and nonlinear properties of semiconductor heterostructures are often governed by excitons that are defined as the bounded state of the electron-hole pair. This is especially important for semiconductors with widebandgap, where the excitonic binding energy is comparable to room temperature. Positively $\left(X^{+}\right)$and negatively $\left(X^{-}\right)$charged excitons, called trions in semiconductor structures, have been the subject of many experimental and theoretical studies in the last years. Positively charges excitons are formed by one electron and two holes, and negatively charged excitons are formed by two electrons and one hole. The theoretical proof of the stability of trions in bulk semiconductors was presented by Lampert [1]. The experimental observation of trions in semiconductor quantum wells (QWs) was achieved in CdTe/CdZnTe [2] and in GaAs/AlGaAs [3 5].

The many-body formalism including Feynman diagram technique was developed to study the collective properties of three-dimensional (3D) trions in the bulk semiconductors [6]. The 3D $X^{+}$and $X^{-}$confined in a semiconductor cylindrical quantum dot were investigated using a variational procedure within the effective mass approximation [7]. The 3D trions in bulk semiconductors [8] and two-dimensional trions [9] were studied theoretically. According to these studies, the 2D trions have binding energies that are larger than the trions in the corresponding bulk materials. The 2D excitons and 2D $X^{-}$in QWs in the presence of $2 \mathrm{D}$ electron gas were observed experimentally by optical measurements of an excitonic recombination line on the photoluminescence spectra from the QW [10]. The experiments devoted to the observation of $2 \mathrm{D} X^{-}$in QWs in magnetic field by studying the magneto-optical absorption spectra were performed in Ref. 22]. Besides, the 2D $X^{-}$in QWs in magnetic field were observed as a results of the analysis of the excitonic recombination line on the electroluminescence spectra from the QW [11]. The experiments devoted to the observation of magneto-optical spectra of $2 \mathrm{D} X^{-}$in QWs were performed [4, 12]. In Ref. [4] the 2D $X^{-}$are studied in coupled quantum wells, where electrons and holes are spatially separated in different QWs.

The problem of finding the wave functions and eigenenergies of $X^{-}$and $X^{+}$formed by three particles is the tree-body problem. There are different approaches were applied to solve three-body problem: the numerical diagonalization of Hamiltonian for the eigenvalue problem, the variational approach, the Hartree-Fock method, the approach of Faddeev equations, the method of the hyperspherical functions (HF). The three-electron quantum dot in a harmonic confinement potential was studied using the HF method [13, 14] and Faddeev equations in the configuration space [15]. The hyperspherical functions method was applied to solve the Schrödinger equation for $2 \mathrm{D} X^{+}$and $X^{-}[16]$. The three-body problem for three electrons in a quantum dot in a magnetic field was solved in the framework of the hyperspherical functions method [17]. The variational method was applied to solve the eigenvalues and eigenfucntions problem for 3D $X^{+}$and $X^{-}$in cylindrical quantum dot [7]. A variational calculation of the ground-state energy of 2D neutral excitons, $X^{+}$, and $X^{-}$in a single QW was performed in Ref. [18]. A variational calculation of the lower singlet and triplet states of positively charged $2 \mathrm{D}$ trions, confined to a single quantum well and in the presence of a perpendicular magnetic field, was performed [19]. The ground-state energy of $2 \mathrm{D} X^{+}$in coupled QWs (CQWs) formed by spatially separated two $2 \mathrm{D}$ heavy holes in one QW and one $2 \mathrm{D}$ electron in neighboring $\mathrm{QW}$ was calculated using 
the variational method within the infinite-hole-mass approximation [20]. The 2D $X^{-}$in the presence of perpendicular magnetic field were studied numerically by the diagonalization of the Hamiltonian in Refs. [21, 22]. The ground state energy of the $2 \mathrm{D} X^{-}$in the presence of $2 \mathrm{D}$ electron gas was calculated using the diagonalization of the eigenvalue problem in the random phase approximation [23]. In Ref. [23] the screening of the Coulomb interaction and the Pauli exclusion principle were considered. A first-principle path integral Monte Carlo study of the binding energy of excitons, 2D positively and negatively charged trions and biexcitons bound to single-island interface defects in 2D GaAs/AlGaAs quantum wells was presented in Ref. 24]. The preliminary results for trions in the system of semiconductor coupled quantum wells were reported at the 21 European Conference on few-body problem in physics [25].

In all theoretical studies mentioned above the Schrödinger equation for the trion was solved numerically using the different methods. In this Paper we propose the type of the trion which wave functions and eigenenergies can be obtained analytically for some conditions. We consider two parallel CQWs. In the case 1 there are electrons in one QW and the electrons and holes in the other QW. In the case 2 there are holes in one QW and the electrons and holes in the other QW. In the case 1 we consider $X^{-}$formed by one electron in one QW and an electron and a hole in the other QW. In the case 2 we consider $X^{+}$formed by one hole in one QW and an electron and a hole in the other QW. The eigenfunction and eigenvalue problem for these trions is the restricted three-body problem, since the motion of the electron (or hole) is restricted in the plane of one QW, and the motion of the electron-hole pair is restricted in the plane of the other QW. We show that for relatively large distance between these CQWs the eigenvalue and eigenfucntion problem for this trions can be solved analytically.

The dilute system of trions at the densities, when the average distance between the trions is much larger than the radius of each trion the system of trions can be treated as the dilute system of the fermions with the pair Coulomb repulsion, which can form non-electronic Wigner crystal. The 3D electron Wigner crystal was described in Ref. 26] and the 2D electron Wigner crystal was described in Ref. 27]. The 2D electron Wigner crystal formed by electrons on films of helium was studied in Refs. [28, 29]. The spectrum of collective excitations for the 2D electron Wigner crystal formed by electrons on a helium film was calculated [29]. The ground state of a 2D electron gas including formation of the 2D Wigner crystal at low densities was calculated using Monte-Carlo method [33]. Wigner crystallization of electrons in 2D quantum dots was analyzed using path integral Monte Carlo approach 34]. We predict the formation of 2D Wigner crystal of trions at the low densities. In our consideration we neglect the influence of the disorder in the quantum wells. We will show that the critical density of the formation of the trion Wigner crystal is sufficiently larger than the critical density of the electron Wigner crystal, since the mass of trion is larger than the mass of the electron.

The Paper is organized in the following way. In Sec. II we introduce the model Hamiltonian for $X^{-}$and $X^{+}$in the CQWs reducing the restricted three-body problem to the $2 \mathrm{D}$ three-body problem for the exciton and the projection of the electron or hole on the plane of the excitonic QW. In Sec. III we review the solution of the Schrödinger for 2D exciton in a single QW. In Sec. IV] we find the wave function of the relative motion of the exciton and electron (hole) and obtain the corresponding energy spectrum. The energy spectrum for the trion, it dependence on the interwell separation and calculation of the conditional probability distribution based on the trion wave functions are presented in Sec. V. The formation of the trion Wigner crystal in the dilute trion gas is discussed in Sec. VI, Finally, our conclusions follow in Sec. VII

\section{THE TOTAL HAMILTONIAN AND WAVE FUNCTION FOR AN ISOLATED TRION}

We consider two parallel coupled quantum wells separated by a spatial barrier of thickness $D$. There are excitons in one QW and electrons or holes in the other QW. One method for obtaining a system of excitons is to create excitons in one region of the QW using laser pumping. 2D electrons or holes can be obtained in the QW by doping semiconductors that tends to create a constant Fermi energy, producing an excess of carriers, either electrons (donors) or holes (acceptors). These excess carriers then give the material either electrons in the conduction bands or holes in the valence band.

Let $r_{i}$ be the coordinates of particles in laboratory frame with effective masses of the electron $m_{e}$ and the hole $m_{h}$. Let's introduce Jacobi coordinates which are an appropriate set for the three-body problem:

$$
\begin{aligned}
\eta & =\left(\mathbf{r}_{2}-\mathbf{r}_{1}\right) \\
\mathbf{r}_{e(h)} & =\left(\mathbf{r}_{3}-\frac{m_{e} \mathbf{r}_{1}+m_{h} \mathbf{r}_{2}}{m_{e}+m_{h}}\right) \\
\mathbf{R} & =\frac{1}{M}\left(m_{e} \mathbf{r}_{1}+m_{h} \mathbf{r}_{2}+m_{e(h)} \mathbf{r}_{3}\right)
\end{aligned}
$$




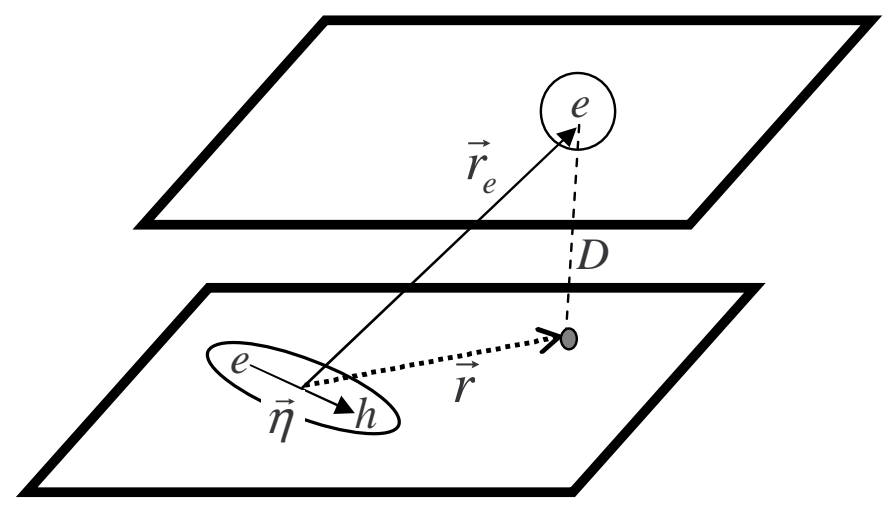

FIG. 1: The restricted three-body problem in the CQWs: an electron in one QW and an electron-hole pair in the other QW.

where $m_{e(h)}$ is the electron (hole) mass, the coordinate vector $\eta$ in 2D space describes of the relative motion of the electron and hole in the exciton with the reduce mass $\mu=m_{e} m_{h} /\left(m_{e}+m_{h}\right)$ in the excitonic QW, the coordinate vector $\mathbf{r}_{e(h)}$ from the center of mass of the exciton to the electron (hole) in the other QW describes the motion of the particles with reduce mass $m=\left(m_{e}+m_{h}\right) m_{e(h)} /\left(m_{e}+m_{h}+m_{e(h)}\right), R$ is the vector of the center of mass of trion and $M=m_{e}+m_{h}+m_{e(h)}$ is the mass of a negatively or positively charged trion.

The total Hamiltonian for an exciton in one QW interacted with an electron (hole) in the other QW is given by

$$
\hat{H}=-\frac{\hbar^{2}}{2 m_{e(h)}} \Delta_{e(h)}+\hat{H}_{e x}+V_{e(h)-e x}
$$

where $V_{e(h)-e x}$ is the potential energy of the interaction between the electron (hole) in one QW and the exciton in an excitonic QW, and $\hat{H}_{e x}$ is the exciton Hamiltonian.

Since the motion of the exciton is restricted in the excitonic QW and the motion of the electron (hole) is restricted in the electronic QW, we replace the coordinate vector of the electron (hole) $\mathbf{r}_{e(h)}$ by its projection $\mathbf{r}$ on plane of the excitonic QW using the relation $r_{e(h)}=\left(r^{2}+D^{2}\right)^{1 / 2}$. Thus, we reduced the restricted 3D three-body problem to the 2D three-body problem on the plane of the excitonic QW as shown in Fig. 1.

The Hamiltonian of the electron and the hole forming the exciton in the excitonic QW has the following form

$$
\hat{H}_{e x}=-\frac{\hbar^{2}}{2 m_{e}} \Delta_{\mathbf{r}_{1}}-\frac{\hbar^{2}}{2 m_{h}} \Delta_{\mathbf{r}_{2}}-\frac{e^{2}}{\epsilon \eta}
$$

where $\Delta_{\mathbf{r}_{1}}$ and $\Delta_{\mathbf{r}_{2}}$ are the Laplacian operators with respect to the components of the vectors $\mathbf{r}_{1}$ and $\mathbf{r}_{2}$ respectively, $\epsilon$ is the dielectric constant, $e$ is the electron charge. The problem of the in-plane motion of interacting electron and hole with effective masses $m_{e}$ and $m_{h}$, respectively, forming the exciton in the excitonic QW can be reduced to that of one particle with reduced mass $\mu$ and energy $E_{e x}$, moving in a Coulomb potential and motion of the center of mass of the exciton.

Let us assume, that the distance between QWs $D$ is greater than the Bohr radius $a_{B}$ of the exciton formed by the bounded state of the electron and the hole in the excitonic QW: $D \gg a_{B}$, where $a_{B}=\hbar^{2} \epsilon /\left(2 m_{e x} e^{2}\right)$. Under this assumption the potential energy $V_{e(h)-e x}$ of the pair interactions between the exciton and electron placed in two parallel quantum wells which is the sum of the Coulomb electron-electron repulsion and electron-hole attraction for the $X^{-}$trion or the hole-hole repulsion and electron-hole attraction for the $X^{+}$trion can be reduced to the polarization energy. Follow Ref. [35] the polarization energy for the interaction of the isolated 2D exciton and an isolated electron can be written in an approximation in the form

$$
V_{e(h)-e x}(r)=-\frac{\alpha}{r_{e(h)}^{4}}=-\frac{\alpha}{\left(r^{2}+D^{2}\right)^{2}}
$$

where $\alpha=\frac{21}{32} \frac{e^{2} a_{B}^{3}}{\epsilon}$ and $r$ is the distance between the center of mass of the exciton and the projection of the electron (hole) on the excitonic plane as shown in Fig. 1. In other words, $\mathbf{r}$ is the projection of the coordinate $\mathbf{r}_{\mathbf{e}(\mathbf{h})}$ defined by Eq. (11) onto the excitonic plane. 
Based on Eqs. (2) - (4) after the separation of the motion of the center of mass of the trion the eigenstates of the trion in coordinates (11) are described by the following Schrödinger equation

$$
\left(-\frac{\hbar^{2}}{2 \mu} \Delta_{\eta}-\frac{e^{2}}{\epsilon \eta}-\frac{\hbar^{2}}{2 m} \Delta_{\mathbf{r}}-\frac{\alpha}{\left(r^{2}+D^{2}\right)^{2}}\right) \Psi(\eta, \mathbf{r})=E \Psi(\eta, \mathbf{r}),
$$

where $\Delta_{\eta}$ and $\Delta_{\mathbf{r}}$ are the Laplacian operators with respect to the components of the vectors $\eta$, and $\mathbf{r}$, respectively. Eq. (5) allows the separation of variables and reduces to the Schrödinger equation that describes the eigenstates of a 2D exciton, and the Schrödinger equation that describes the relative motion of the exciton and electron or exciton and hole in the 2D excitonic plane.

Seeking the solution of (5) in the form $\Psi(\eta, \mathbf{r})=\Psi_{e x}(\eta) \Psi_{e(h)}(\mathbf{r})$ and using the separation of variables we obtain

$$
\begin{gathered}
\left(-\frac{\hbar^{2}}{2 \mu} \Delta_{\eta}-\frac{e^{2}}{\epsilon \eta}\right) \Psi_{e x}(\eta)=E_{e x} \Psi_{e x}(\eta) \\
\left(-\frac{\hbar^{2}}{2 m} \Delta_{\mathbf{r}}-\frac{\alpha}{\left(r^{2}+D^{2}\right)^{2}}\right) \Psi_{e(h)}(\mathbf{r})=E_{e(h)} \Psi_{e(h)}(\mathbf{r}) .
\end{gathered}
$$

Therefore, the energy spectrum of the trion is

$$
E=E_{e x}+E_{e(h)}
$$

Let us consider (6) and (7) separately.

\section{THE WAVE FUNCTION AND ENERGY SPECTRUM OF THE 2D EXCITON}

The eigenstates of the electron and the hole forming a 2D exciton in the excitonic QW are described by the Schrödinger (6), that represents a two-body problem which is like the two-dimensional hydrogenic problem. Below we follow the approach of Ref. [36] for finding the wavefunctions and eigenenergies of the 2D hydrogen atom in polar coordinates for the arbitrary values of two masses. The Schrödinger equation (6) that describes the relative $2 \mathrm{D}$ motion of an electron and hole, in polar coordinates has the form

$$
\left[-\frac{\hbar^{2}}{2 \mu}\left(\frac{\partial^{2}}{\partial \eta^{2}}+\frac{1}{\eta} \frac{\partial}{\partial \eta}+\frac{1}{\eta^{2}} \frac{\partial^{2}}{\partial \varphi^{2}}\right)-\frac{e^{2}}{\epsilon \eta}\right] \Psi_{e x}(\eta, \varphi)=E_{e x} \Psi_{e x}(\eta, \varphi) .
$$

After the standard separation of the variables $\eta$ and $\varphi$ we obtain the radial Schrödinger equation and the solution for the 2D exciton wave function in terms of associated Laguerre polynomials is presented in [36], and the exciton spectrum is given by

$$
E_{n} \equiv E_{e x}=-\frac{\mu e^{4}}{2 \epsilon^{2}(n-1 / 2)^{2} \hbar^{2}}
$$

where $n=1,2,3, \ldots$ are the quantum numbers. We can see that in the ground state at $n=1$ the binding energy of the exciton is $E_{1}=2 \mu e^{4} /\left(\epsilon^{2} \hbar^{2}\right)$, and the characteristic radius of the exciton corresponding to the wave function $\Psi_{e x}(\eta)$ at $n=1, l=0$ is given by $a_{B}=\hbar^{2} \epsilon /\left(2 \mu e^{2}\right)$. Interestingly enough that by replacing $(n-1 / 2)$ by $n$ retrieves relevant formulas of the $3 \mathrm{D}$ case, particularly quantization conditions (10).

\section{THE WAVE FUNCTION AND ENERGY OF THE RELATIVE MOTION OF THE EXCITON AND ELECTRON AND EXCITON AND HOLE}

The eigenstates of the electron (hole) in the field of the exciton are described by the Schrödinger (77), which represents a motion of the projection of the electron (hole) on the plane of the excitonic QW. Using the polar coordinates $\mathbf{r}$ and 
$\phi$ and substituting $\Psi_{e(h)}(r, \phi)=r^{-1 / 2} u_{e(h)}(r, \phi)$ into Eq. (7), we obtain the 2D Schrödinger equation of the relative motion of an exciton and an electron (hole)

$$
\left[-\frac{\hbar^{2}}{2 m}\left(\frac{\partial^{2}}{\partial r^{2}}+\frac{1}{4 r^{2}}+\frac{1}{r^{2}} \frac{\partial^{2}}{\partial \phi^{2}}\right)+V_{e(h)-e x}(r)\right] u_{e(h)}(r, \phi)=E_{e x} u_{e(h)}(r, \phi),
$$

where $V_{e(h)-e x}(r)$ is given by Eq. (4). The $2 \mathrm{D}$ motion of the projection of the electron (hole) in the plane of the excitonic QW, can be treated as that of a single particle with reduced mass $m$ and energy $E_{e(h)}$, moving in a potential $\frac{\alpha}{\left(r^{2}+D^{2}\right)^{2}}$. Assuming $r \ll D$, the Taylor expansion of $V_{e(h)-e x}(r)$ from Eq. (4) up to the linear term results in

$$
V_{e(h)-e x}(r)=-\frac{\alpha}{D^{4}}+\frac{1}{2} \frac{\alpha}{D^{6}} r^{2} .
$$

Using potential (12) in (11) we obtain the Schrödinger's equation that describes the two-dimensional motion of a single electron (hole), that corresponds to the 2D harmonic oscillator with the harmonic parabolic potential $\gamma \frac{r^{2}}{2}$, where $\gamma=\alpha / D^{6}$, when the energy is counted from the energy level $E_{e(h)}^{(0}=-\alpha / D^{4}$.

Using the standard separation of the variables $r$ and $\phi$ in and follow Refs. [37, 38] we obtain the radial Schrödinger equation and the solution for the eigenfunctions for the projection of the electron (hole) on the plane of the excitonic QW can be written in terms of associated Laguerre polynomials. The characteristic radius $a=(\hbar /(2 \sqrt{m \gamma}))^{1 / 2}$ of the wave function is directly proportional to $D^{3 / 2}\left(a \sim a_{B}^{-1 / 2} D^{3 / 2}\right)$. The corresponding energy spectrum is given by

$$
E_{N L} \equiv E_{e(h)}=-\frac{\alpha}{D^{4}}+(2 N+1+|L|) \hbar\left(\frac{\alpha}{D^{6} m}\right)^{1 / 2} .
$$

where $N=\min \left(\widetilde{n}, \widetilde{n}^{\prime}\right), L=\widetilde{n}-\widetilde{n}^{\prime}, \widetilde{n}, \widetilde{n}^{\prime}=0,1,2,3, \ldots$ are the quantum numbers.

As it seen from Eq. (13), the first term is proportional to $D^{-4}$, and the second term is proportional to $D^{-3}$. Therefore, for the some values of the separation distance $D$ between the QW the energy becomes positive. That means that at the interwell separations greater than some critical $D_{c r}, D>D_{c r}$ the energy $E_{N L}$ is positive, $E_{N L}>0$, and at $D<D_{c r}$, the energy $E_{N L}$ is negative, $E_{N L}<0$. For example, at the lowest quantum state $N=L=0$ as it follows from Eq. (13) the ground state energy for the electron (hole) in the parabolic well of the exciton in the excitonic QW is given by

$$
E_{00}=-\frac{\alpha}{D^{4}}+\hbar\left(\frac{\alpha}{D^{6} m}\right)^{1 / 2} .
$$

Using Eq. (14), and the value for $\alpha$ we obtain the critical interwell separation $D_{c r}$ :

$$
D_{c r}=\left(\frac{21}{32}\right)^{1 / 2} \frac{e a_{B}^{3 / 2} m^{1 / 2}}{\hbar \epsilon^{1 / 2}} .
$$

If we consider the trions in GaAs/AlGaAs CQWs, then we have $m_{e}=0.07 m_{0}, m_{h}=0.15 m_{0}, \epsilon=13$, where $m_{0}$ is the free electron mass. Using Eq. (15) for the GaAs/AlGaAs CQWs we obtain for $X^{-}$the critical interwell separation $D_{c r}=4.4 \mathrm{~nm}$, and for $X^{+}$we find $D_{c r}=5.6 \mathrm{~nm}$. However, let us mention that our approach is valid only for $D \gg D_{c r}$, because the condition of the Taylor expansion of the electron (hole)-exciton interaction potential energy is $D \gg a_{B}$.

\section{DISCUSSION OF THE RESULTS FOR THE SINGLE TRION}

In our cluster approach, when the trion is the system of interacting exciton and electron or hole, the wavefunction of the trion $\Psi(\eta, \mathbf{r})$ is a product of the wavefunctions of the exciton and relative motion of exciton and electron (hole) and, therefore, can be expressed in terms of associated Laguerre polynomials. As it follows from (8) the energy spectrum of the trion is defined by Eqs. (10) and (13) and is given by

$$
E_{n N L}=-\frac{21}{32} \frac{e^{2} a_{B}^{3}}{\epsilon D^{4}}-\frac{\mu e^{4}}{2 \epsilon^{2}(n-1 / 2)^{2} \hbar^{2}}+(2 N+1+|L|) \hbar\left(\frac{21}{64} \frac{e^{2} a_{B}^{3}}{\epsilon m D^{6}}\right)^{1 / 2} .
$$

The energy spectrum of the trion corresponds to the discrete levels corresponding to the bounded states of the electron (or hole) and exciton. Therefore, the trion wavefunction represents the bounded state with the characteristic 

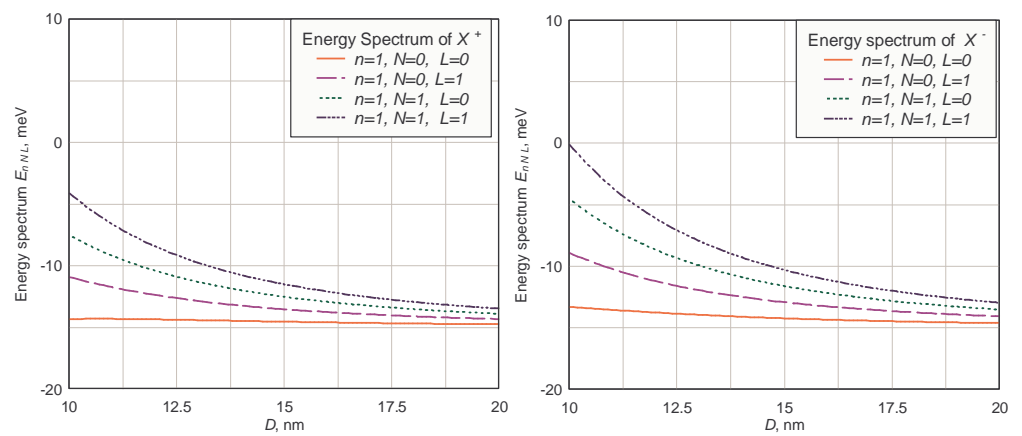

FIG. 2: The energy spectrum for $X^{+}$and $X^{-}$as a function of the interwell separation $D$ for different quantum states.

radius at large $D$ corresponding to the radius of the trion $a_{t}=a$. Since $a$ is directly proportional to $D^{3 / 2}$, which is the highest degree of $D$ compare to the other characteristic radiuses, thus the radius of the trion is proportional to $D^{3 / 2}$. The calculated energy spectrum as a function of the interwell separation $D$ for different quantum numbers for $X^{+}$and $X^{-}$are shown in Fig. 2 .

TABLE I: The energy spectrum of $X^{+}$and $X^{-}$trions for interwell separation $D=12 \mathrm{~nm}$ for different quantum states.

\begin{tabular}{|c|c|c|c|c|c|c|c|}
\hline$n$ & $N$ & $L X^{+}$, & Energy, meV & & $N$ & $L$ & nergy, meV \\
\hline 1 & 0 & 0 & -14.7 & 1 & 0 & 0 & -14.1 \\
\hline 1 & 0 & 1 & -12.7 & 1 & 0 & 1 & -11.6 \\
\hline 1 & 0 & 2 & -10.8 & 1 & 0 & 2 & -9.0 \\
\hline 1 & 0 & 3 & -8.8 & 1 & 0 & 3 & -6.5 \\
\hline 1 & 0 & 4 & -6.8 & 1 & 0 & 4 & -3.9 \\
\hline 1 & 0 & 5 & -4.8 & 1 & 0 & 5 & -1.3 \\
\hline 1 & 0 & 6 & -2.9 & & & & \\
\hline 1 & 0 & 7 & -0.9 & & & & \\
\hline 1 & 1 & 0 & -10.8 & & 1 & 0 & -9.8 \\
\hline 1 & 1 & 1 & -8.8 & 1 & 1 & 1 & -6.5 \\
\hline 1 & 1 & 2 & -6.8 & 1 & 1 & 2 & -3.9 \\
\hline 1 & 1 & 3 & -4.8 & 1 & 1 & 3 & -1.3 \\
\hline 1 & 1 & 4 & -2.8 & & & & \\
\hline 1 & 1 & 5 & -0.9 & & & & \\
\hline 1 & 2 & 0 & -6.8 & & 2 & 0 & -3.9 \\
\hline 1 & 2 & 1 & -4.8 & & 2 & 1 & -1.3 \\
\hline 1 & 2 & 2 & -2.9 & & & & \\
\hline 1 & 2 & 3 & -0.9 & & & & \\
\hline 1 & 3 & 0 & -2.9 & & & & \\
\hline 1 & 3 & 1 & -0.9 & & & & \\
\hline 2 & 0 & 0 & -1.0 & 2 & 0 & 0 & -0.5 \\
\hline
\end{tabular}

Eq. (16) shows the dependence of the energy spectrum of a trion on the interwell separation $D$. Typical thickness of the barrier between two parallel QWs $D$ varies from one experiment to the other experiment. The thickness of barrier separation for production of indirect excitons was $4 \mathrm{~nm}$ [39, 40]. However, for interwell distances used in the drag experiments in Ref. [41] are $D=17.5 \mathrm{~nm}$ and $D=22.5 \mathrm{~nm}$, and the interwell separations used in the drag experiment in Ref. [42] are $20 \mathrm{~nm}$ and $30 \mathrm{~nm}$. Therefore, we can conclude that it is possible to experimentally construct the QWs 
with thickness of the barrier from $4 \mathrm{~nm}$ to $30 \mathrm{~nm}$. In our calculations we vary the interwell separation within these limits (from $10 \mathrm{~nm}$ to $20 \mathrm{~nm}$ ). Analysis of the energy spectrum of the trion, for example for $D=12 \mathrm{~nm}$, shows that $X^{+}$exists when the exciton is in the ground state with $n=1$ and the hole is in states: $N=0, L=0,1, \ldots, 7$ or the exciton is in state $n=2$ and the hole is in the ground state. $X^{-}$exists when the exciton is in the ground state and the electron is in states: $N=0, L=0,1, \ldots, 5$ or the exciton is in the state $n=2$ and the hole is in the ground state. Let us once again mention that there is the critical interwell separations when for $D \geq D_{c r}$ the the ground state energy of the trion is negative, and if we consider the trions in GaAs/AlGaAs coupled QWs, then we obtain for $X^{-}$the critical interwell separation $D_{c r}=4.4 \mathrm{~nm}$, and for $X^{+}$we find $D_{c r}=5.6 \mathrm{~nm}$. However, our approach is valid only for $D \gg D_{c r}$, because the condition of the Taylor expansion of the electron (hole)-exciton interaction potential energy is $D \gg a_{B}$.

It is interesting to mention that from Eq. (16) follows that, for example, for $L=0$ the maximum value of $N=3$ for distance $D=12 \mathrm{~nm}$ and $N=7$ for $D=15 \mathrm{~nm}$ when the exciton is in ground state and the energy of the trion is still negative. The energies spectrum of $X^{+}$and $X^{-}$trions for the interwell separation $D=12 \mathrm{~nm}$ for different quantum numbers are presented in the Table I.

Analysis of Table I shows that the $X^{-}$trion does not exist in the states $(n, N, L)=$ $(1,0,6),(1,0,7),(1,1,4),(1,1,5)),(1,2,2),(1,2,3),(1,3,0),(1,3,1)$, while the the $X^{+}$trion exists in all these states. It is clear from Eq. (16) that the energy spectrum depends on the trion reduce mass $m$. The reduced mass for the $X^{+}$trion is $0.89 m_{0}$ and it is greater than one for the $X^{-}$trion, which is $0.53 m_{0}$, because the effective hole mass $m_{h}$ is greater than the effective electron mass $m_{e}$, and, therefore, the second positive term for the trion energy in Eq. (16) is greater for $X^{-}$than for $X^{+}$, since the second term is proportional to $m^{-1 / 2}$. Therefore, for the states listed above, the binding energy for $X^{-}$is positive, and the bound state for $X^{-}$does not exist, while for the same quantum states the binding energy for $X^{+}$is negative, and the bound state for $X^{+}$exists.

The structure of the wave function of the trion which is a product of the wavefunctions of the exciton and relative motion of exciton and electron (hole) can be tested by calculation of the conditional probability distribution (CPD) which is the pair correlation function $P\left(\mathbf{r}, \mathbf{r}_{\mathbf{0}}\right)$ that expresses the probability of finding an electron or hole at the position $r$ given when the exciton is located at the position $r_{0}$. Therefore, the CPD gives us information on when exciton located at $r_{0}$ sees an electron (hole) at $r$ and defined as [30 32]

$$
P\left(\mathbf{r}, \mathbf{r}_{\mathbf{0}}\right)=\frac{\left\langle\Psi\left|\sum_{i \neq j}^{N} \delta\left(\mathbf{r}_{i}-\mathbf{r}\right) \delta\left(\mathbf{r}_{j}-\mathbf{r}_{0}\right)\right| \Psi\right\rangle}{\langle\Psi \mid \Psi\rangle} .
$$

In Eq. (17) $\Psi$ is the wave function of the trion, which is a product of the wavefunctions of the exciton and relative motion of exciton and electron (hole). We use the accurate analytical wave functions for the trion to compute the CPDs. In Fig. 3 the CPDs are displayed for the state $n=1, l=0, N=2, L=0$ for the different interwell separations that vary from $D=10 \mathrm{~nm}$ to $D=18 \mathrm{~nm}$. At the interwell separation $D=10 \mathrm{~nm}$ the confinement potential is strong and the contribution of the single-particle energies to the total energy of the trion is larger. The hole is confined in a rather compact region so that the CPD does not show clear two peaks. On the contrary, for $D=14 \mathrm{~nm}$ the effect of the confinement becomes weaker. The size of the system grows and we see clearly well separated hump structure in Fig. [3r and Fig. 3 3 . For the comparison in Fig. 4 the CPDs for $X^{+}$trion is presented when the hole is in the excited state with $N=3$ for different interwell separations. The CPDs for four specific states i.e., the $n=1, l=0, N=1$, $L=0$, the $n=1, l=0, N=1, L=1$, the $n=1, l=0, N=1, L=2$ and $n=1, l=0, N=1, L=3$ when the interwell separation $D=14 \mathrm{~nm}$ in conjunction with identification of regularities related to the quantum number $L$ are shown in Fig. 5 . By examining the CPD's associated with selected states one can make a conclusion on the dependence of the CPD on the quantum number $L$.

\section{TRION WIGNER CRYSTAL}

It is well known that the dilute system of the electrons can form the 2D Wigner crystal [33]. Based on the existence of the trions in the CQWs we can propose that trions form of 2D Wigner crystal at the low densities alike to the 2D Wigner crystal formed by the dilute system of the electrons. The dilute system of trions, when the average distance between the trions is much larger than the radius of each trion, can be treated as the dilute system of the fermions with the pair Coulomb repulsion, that undergo a phase transition and crystallize by forming the Wigner crystal. To minimize the potential energy, the trions experiencing Coulomb repulsion form a triangular lattice in 2D system. In other words, this is an example when Wigner crystal phase is occurring in non-electronic system at low density.

The Wigner crystal formed by the composite particles was studied in several recent publications. Ref. [43] was devoted to Wigner crytallization of the dipole cold atoms, while Wigner crystallization of dipole indirect excitons in 

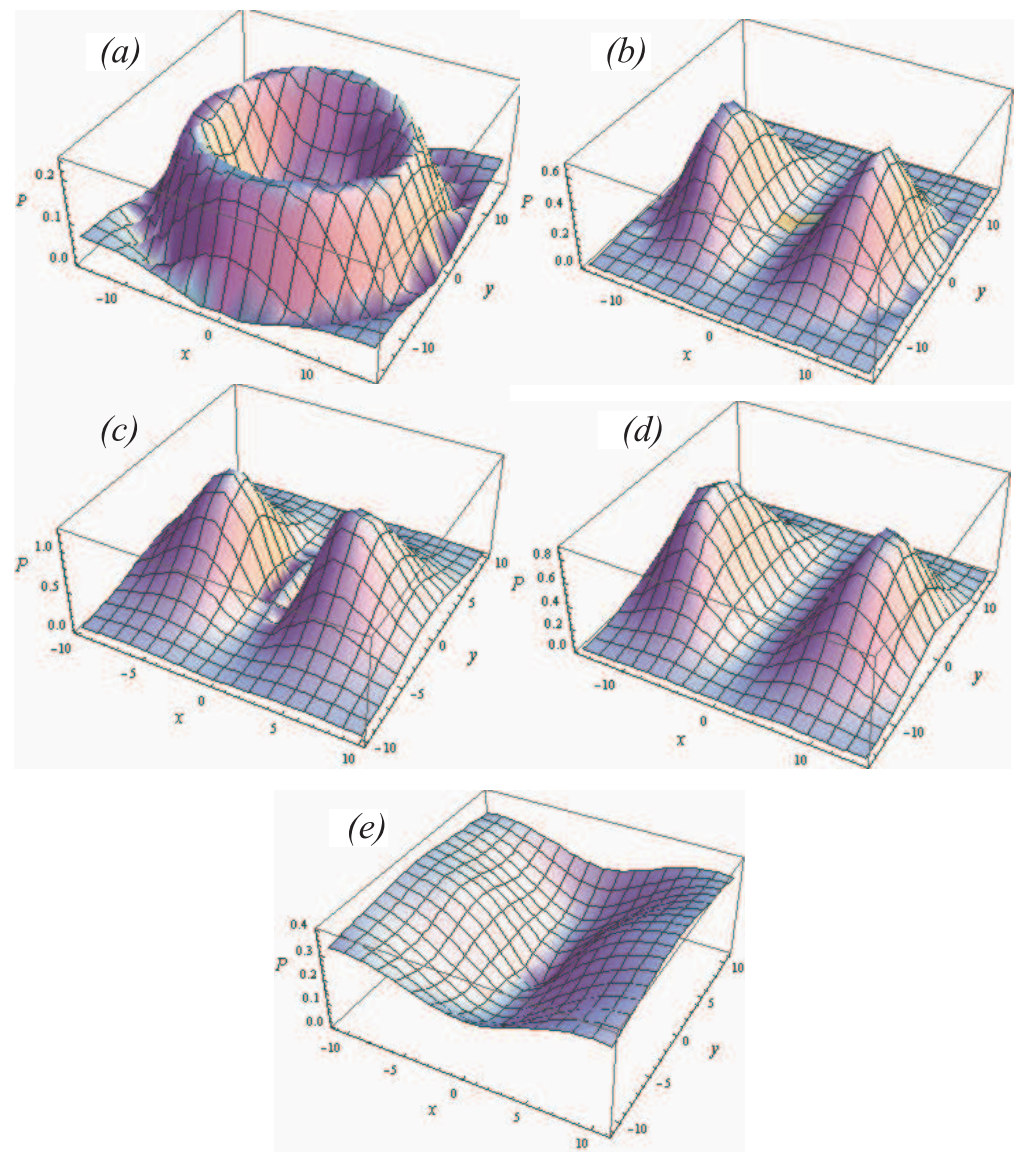

FIG. 3: The CPDs for $X^{+}$trion for the state $n=1, l=0, N=2, L=0$ and different inrewell separations: (a) $D=10 \mathrm{~nm}$; (b) $D=12 \mathrm{~nm}$; (c) $D=14 \mathrm{~nm}$; (d) $D=16 \mathrm{~nm}$; (e) $D=18 \mathrm{~nm}$.
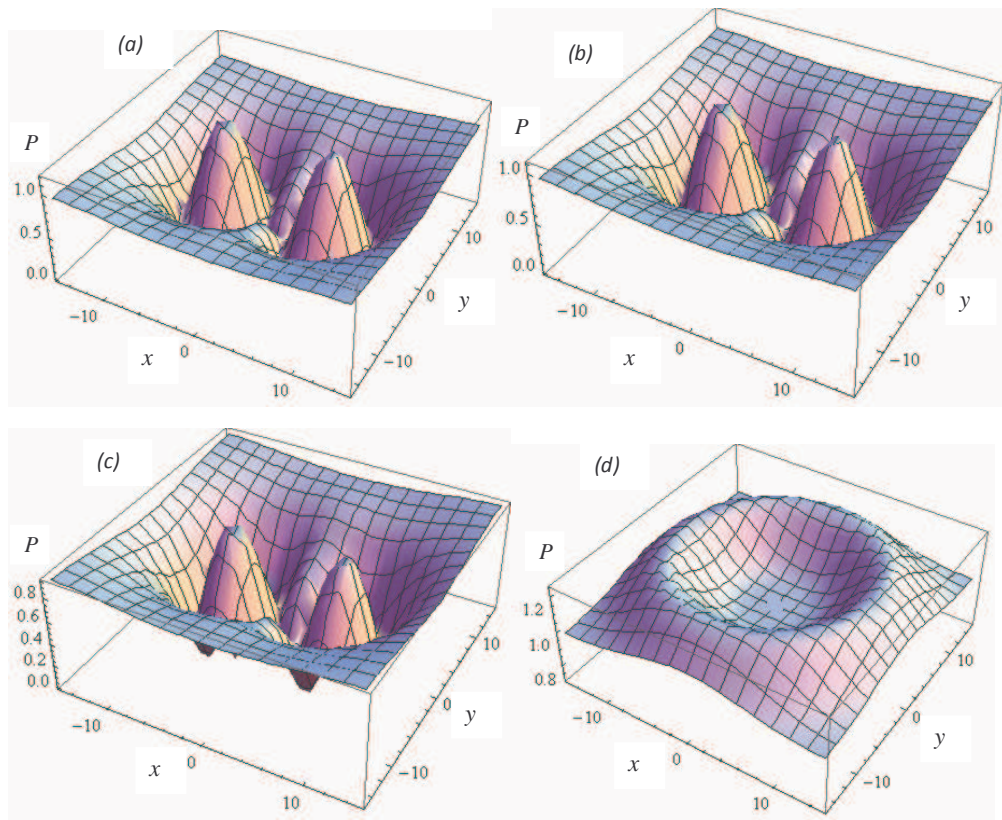

FIG. 4: The CPDs for $X^{+}$trion for the state $n=1, l=0, N=3, L=0$ and different inrewell separations: (a) $D=12$ nm; (b) $D=14 \mathrm{~nm}$; (c) $D=16 \mathrm{~nm}$; (d) $D=18 \mathrm{~nm}$. 

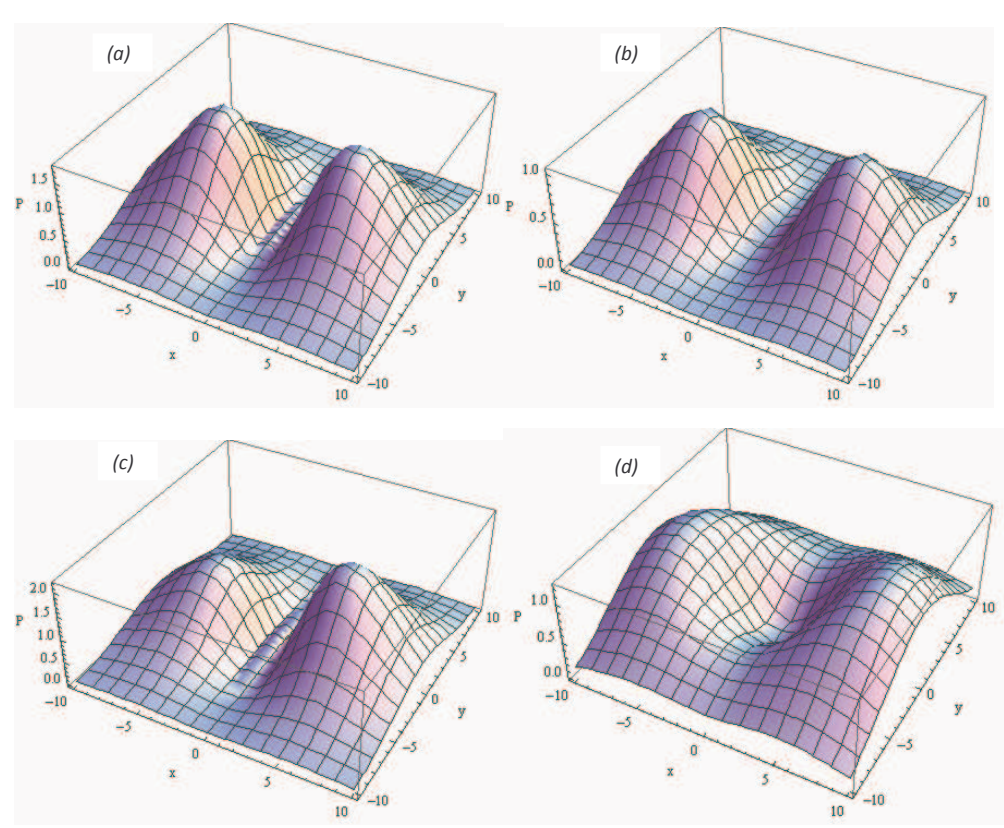

FIG. 5: The CPDs for $X^{+}$trion when interwell separation $D=14 \mathrm{~nm}$ for the states: (a) $n=1, l=0, N=1, L=0 ;$ (b) $n=1, l=0, N=1, L=1$; (c) $n=1, l=0, N=1, L=2$; (d) $n=1, l=0, N=1, L=3$.

QWs was studied in Refs. [44 46]. Wigner crystallization of the dipole cold atoms in magnetic field was considered in Ref. [45]. The Hamiltonian of the trions in CQWs will be written alike to the Hamiltonian of composite dipole particles in the papers listed above, when the energy of the composite particles is counted relative to the binding energy of the composite dipole particle. The total Hamiltonian $\hat{H}_{t o t}$ per unit area of the interacting system of $\tilde{N}$ trions per unit area is similar to that for the electrons [33]:

$$
\hat{H}_{t o t}=\hat{K}+\hat{U} .
$$

In Eq. (18) $\hat{K}$ is the kinetic energy of the centers mass of the trions given by

$$
\hat{K}=\frac{\hbar^{2}}{2 m_{+(-)} S} \sum_{i=1}^{\tilde{N}} \Delta_{\mathbf{R}_{i}},
$$

where $S$ is the area of the system, $M_{-}=m_{e}+m_{h}+m_{e}$ is the mass of the trion $X^{-}$and $M_{+}=m_{e}+m_{h}+m_{h}$ is the mass of the trion $X^{+}$, and $\hat{U}$ is the potential energy of the trion-trion Coulomb repulsion per unit area:

$$
\hat{U}=\frac{e^{2}}{\epsilon S} \sum_{1 \leq i<j \leq \tilde{N}} \frac{1}{\left|\mathbf{R}_{i}-\mathbf{R}_{j}\right|} .
$$

In Eq. (20) $\mathbf{R}_{i}$ and $\mathbf{R}_{j}$ are coordinates of the centers of the mass of the trions. Let us mention that in the dilute system of trions when the average distance between the trions is much larger than the radius of each trion, we can neglect the interaction between excitons of different trions and exciton in one trion and electron (or hole) in the other neighbored trion, which are proportional $1 / r^{6}$ (see Ref. [47]) and $1 / r^{4}$ (see Eq. (44)), respectively, compare to the Coulomb repulsion between the electrons (or holes) in the different trions. In this approximation we replace the Coulomb repulsion between the electrons (or holes) in different trions by the Coulomb repulsion between the centers of mass of different trions. Therefore, we can treat the the dilute system of the charged spin- $1 / 2$ fermions interacting with the pair Coulomb repulsion formed by the trions the same way as the dilute system of electrons.

According to Ref. [48], the qualitative criterion of the stability of the Wigner crystal is the condition, when the potential energy dominates the kinetic energy: $\langle\hat{U}\rangle>\langle\hat{K}\rangle$, where $\langle\hat{U}\rangle$ is the average potential energy per unit area, and $\langle\hat{K}\rangle$ is the average value of the kinetic energies of the centers of mass of trions per unit area. This allows to determine the density at which the trion system becomes a Wigner crystal. Let us mention that the averaging of $\hat{U}$ and $\hat{K}$ is calculated as the averaging by the many-particle antisymmetric wave function of the centers of mass of trions which is similar to the antisymmetric many-electron wave function [33]. 
Let's show that at zero temperature trion Wigner crystal exist at higher densities than electron (or hole) Wigner crystal. Estimating $\hat{U}$ and $\hat{K}$ for the trion system analogously to the electron system [33], we conclude that the 2D trion Wigner crystal is stable when the dimensionless density parameter $r_{s} \geq 37$ at $T=0$. The so-called Wigner-Seitz radius is defined as $r_{s}=a / a_{0}$, where $a=(\pi \rho)^{-1 / 2}$ is the average distance between the centers of mass of trions, $\rho$ is the $2 \mathrm{D}$ density of trions, and $a_{0}=\hbar^{2} \epsilon /\left(M_{+(-)} e^{2}\right)$. The last condition enables us to determine the density at which the trion gas becomes a Wigner crystal. For the negative trions $X^{-}$this condition corresponds to $\rho \leq 4.14 \times 10^{13} \mathrm{~m}^{-2}$ and for the positive trions $X^{+} \rho \leq 6.75 \times 10^{13} \mathrm{~m}^{-2}$. For the electron Wigner crystal we substitute $a_{0}=\hbar^{2} \epsilon /\left(m_{e} e^{2}\right)$, which results in $\rho \leq 2.41 \times 10^{12} \mathrm{~m}^{-2}$. For the hole Wigner crystal we substitute $a_{0}=\hbar^{2} \epsilon /\left(m_{h} e^{2}\right)$, which results in $\rho \leq 1.11 \times 10^{13} \mathrm{~m}^{-2}$. Therefore, the trion Wigner crystal can be formed at the sufficiently higher densities than electron (or hole) Wigner crystal, because the mass of trion is greater than the mass of the electron or hole.

Above we considered the trion gas at zero temperature. At finite temperatures the defects will always destroy the long-range translational order in the 2D Wigner crystals. We assume that a $2 \mathrm{D}$ Wigner crystal in the ground state will have no defects. Also let us mentioned that in practice, it is difficult to experimentally realize a Wigner crystal because quantum mechanical fluctuations overpower the Coulomb repulsion and quickly cause disorder. A low dense system is needed.

\section{CONCLUSIONS}

In this Paper we have reduced the three-body restricted problem for a spatially separated exciton and electron (hole) located in the CQWs to the 2D three-body problem of the exciton and the projection of the electron (hole) on the plane of the exciton QW. In the limit of large spatial separation between the electron (hole) and exciton QWs we have obtained the analytical expression for the wave functions of the trions $X^{-}$and $X^{+}$with the spatially separated electron (hole) and exciton. Analytical results for the energy spectrum of the trion formed by the exciton and electron (hole) separated in different quantum wells are obtained, its dependence on the interwell separations is analyzed and a conditional probability distribution is calculated. The differences in the binding energies for $X^{+}$and $X^{-}$due to the difference between electron and hole masses is analyzed. The 2D Wigner crystallization of the trions in the CQWs is predicted. Due to the fact that mass of the trion is greater than the mass of electron, the critical density of the formation of the trion Wigner crystal at zero temperature is sufficiently greater than the critical density of the electron Wigner crystal.

\section{Acknowledgments}

O. L. B. and R. Ya. K. were supported by PSC CUNY grant 63443-00 41. O. L. B. and R. Ya. K. are thankful to Boris Altshuler and Yurii Rubo for the useful discussions.

[1] M. A. Lampert, Phys. Rev. Lett. 1, 450 (1958).

[2] K. Kheng, R. T. Cox, Y. M. d'Aubigne, F. Bassani, K. Saminadayar, and S. Tatarenko, Phys. Rev. Lett. 71, 1752 (1993).

[3] G. Finkelstein, H. Shtrikman, and I. Bar-Joseph, Phys. Rev. B 53, R1709 (1996); 53, 12593 (1996).

[4] A. J. Shields, J. L. Osborne, D. M. Whittaker, M. Y. Simmons, M. Pepper, and D. A. Ritchie, Phys. Rev. B 55, 1318 (1997).

[5] A. J. Shields, F. M. Bolton, M. Simmons, M. Pepper, and D. A. Ritchie, Phys. Rev. B 55, R1970 (1997).

[6] M. Combescot, O. Betbeder-Matibet and F. Dubin, Eur. Phys. J. B 42, 63 (2004).

[7] S. A. Safwan, M. H. Hekmat, A. S. Asmaa, and N. Elmeshed, Physica E 41, 150 (2008).

[8] G. Munschy and B. Stébé, Phys. Status Solidi B 64, 213 (1974).

[9] B. Stébé and A. Aniane, Superlattices Microstruct. 5, 545 (1989).

[10] G. Finkelstein, H. Shtrikman, and I. Bar-Joseph, Phys. Rev. Lett. 74, 976 (1995).

[11] H. Buhmann, L. Mansouri, J. Wang, P. H. Beton, N. Mori, L. Eaves, M. Henini, and M. Potemski, Phys. Rev. B 51, 7969 (1995).

[12] A. J. Shields, M. Pepper, D. A. Ritchie, M. Y. Simmons, G. A. C. Jones, Phys. Rev. B 51, 18049 (1995).

[13] N. F. Johnson and L. Quiroga, Phys. Rev. Lett. 74, 427 (1995).

[14] W. Y. Ruan and H. F. Cheung, J. Phys.: Condens. Matter 9, 10901 (1997).

[15] M. Braun and O. I. Kartavtsev, Nucl. Phys. A 698519 (2001).

[16] W. Y. Ruan, K. S. Chan, H. P. Ho, R. Q. Zhang, and E. Y. B. Pun, Phys. Rev. B 60, 5714 (1999).

[17] R. Ya. Kezerashvili, L. L. Margolin, and Sh. M. Tsiklauri, Few Body Syst. 44, 241 (2008). 
[18] C. Riva, F. M. Peeters, and K. Varga, Phys. Rev. B 61, 13873 (2000).

[19] C. Riva, F. M. Peeters, and K. Varga, Phys. Rev. B 64, 235301 (2001).

[20] R. A. Sergeev and R. A. Suris, Semiconductors 37, 1205 (2003).

[21] J. R. Chapman, N. F. Johnson, V. N. Nicopoulos, Phys. Rev. B 55, R10221 (1997).

[22] D. M. Whittaker and A. J. Shields, Phys. Rev. B 56, 15185 (1997).

[23] L. C. O. Dacal and J. A. Brum, Physica E 12546 (2002).

[24] A. V. Filinov, C. Riva, F. M. Peeters, Yu. E. Lozovik, and M. Bonitz, Phys. Rev. B 70, 035323 (2004).

[25] O. L. Berman and R. Ya. Kezerashvili, Few Body Syst. 50, 407 (2011).

[26] D. Pines, Elementary Excitations in Solids (W. A. Benjamin: N.Y., 1963).

[27] L. Bonsall and A. A. Maradudin, Phys. Rev. B 15, 1959 (1977).

[28] F. M. Peeters and P. M. Platzman, Phys. Rev. Lett. 50, 2021 (1983).

[29] F. M. Peeters, Phys. Rev. B 30, 159 (1984).

[30] C. Yannouleas and U. Landman, Phys. Rev. Lett. 85, 1726 (2000).

[31] C. Yannouleas and U. Landman, Phys. Rev. B 70, 235319 (2004).

[32] C.Yannouleas and U. Landman, Phys. Stat. Sol. (a) 203, 1160 (2006).

[33] B. Tanatar and D. M. Ceperley, Phys. Rev. B 39, 5005 (1989).

[34] A. V. Filinov, M. Bonitz, and Yu. E. Lozovik, Phys. Rev. Lett. 86, 3851 (2001).

[35] Yu. E. Lozovik and M. V. Nikitkov, JETP 89, 775 (1999).

[36] B. Zaslow and M. E. Zandler, Am. J. Phys. 35, 1118 (1967).

[37] P. A. Maksym and T. Chakraborty, Phys. Rev. Lett. 65, 108 (1990).

[38] A. Iyengar, J. Wang, H. A. Fertig, and L. Brey, Phys. Rev. B 75, 125430 (2007).

[39] L. V. Butov, C. W. Lai, A. L. Ivanov, A. C. Gossard, D. S. Chemla, Nature 417, 47 (2002).

[40] L. V. Butov, A. C. Gossard, and D. S. Chemla, Nature 418, 751 (2002).

[41] T. J. Gramila, J. P. Eisenstein, A. H. MacDonald, L. N. Pfeiffer, and K. W. West, Phys. Rev. Lett. 66, 1216 (1991).

[42] J. A. Seamons, C. P. Morath, J. L. Reno, and M. P. Lilly, Phys. Rev. Lett. 102, 026804 (2009).

[43] C. Mora, O. Parcollet, and X. Waintal, Phys. Rev. B 76, 064511 (2007).

[44] Yu. E. Lozovik, S. Yu. Volkov, and M. Willander, JETP Letters 79, 473 (2004).

[45] G. E. Astrakharchik, J. Boronat, I. L. Kurbakov, and Yu. E. Lozovik, Phys. Rev. Lett. 98, 060405 (2007).

[46] K. Sperlich, P. Ludwig, A. Filinov, M. Bonitz, H. Stolz, D. Hommel, and A. Gust, Phys. Stat. Sol. (c) 6, 551 (2009).

[47] O. L. Berman and Yu. E. Lozovik, JETP 84, 1027 (1997).

[48] P. M. Platzman and H. Fukuyama, Phys. Rev. B 10, 3150 (1974). 